\title{
Esophageal electrode model and simulation of directed transesophageal electrical pacing of the spinal cord
}

\begin{abstract}
Spinal cord stimulation (SCS) is the most commonly used technique of neurostimulation. It involves the stimulation of the spinal cord and is therefore used to treat chronic pain. The existing esophageal catheters are used for temperature monitoring during an electrophysiology study with ablation and transesophageal echocardiography. The aim of the study was to model the spine and new esophageal electrodes for the transesophageal electrical pacing of the spinal cord, and to integrate them in the Offenburg heart rhythm model for the static and dynamic simulation of transesophageal neurostimulation. The modeling and simulation were both performed with the electromagnetic and thermal simulation software CST (Computer Simulation Technology, Darmstadt). Two new esophageal catheters were modelled as well as a thoracic spine based on the dimensions of a human skeleton. The simulation of directed transesophageal neurostimulation is performed using the esophageal balloon catheter with an electric pacing potential of $5 \mathrm{~V}$ and a trapezoidal signal. A potential of $4.33 \mathrm{~V}$ can be measured directly at the electrode, $3.71 \mathrm{~V}$ in the myocardium at a depth of $2 \mathrm{~mm}, 2.68 \mathrm{~V}$ in the thoracic vertebra at a depth of $10 \mathrm{~mm}$, $2.1 \mathrm{~V}$ in the thoracic vertebra at a depth of $50 \mathrm{~mm}$ and $2.09 \mathrm{~V}$ in the spinal cord at a depth of $70 \mathrm{~mm}$. The relation between the voltage delivered to the electrodes and the voltage applied to the spinal cord is linear. Virtual heart rhythm and catheter models as well as the simulation of electrical pacing fields and electrical sensing fields allow the static and dynamic simulation of directed transesophageal electrical pacing of the spinal cord. The 3D simulation of the electrical sensing
\end{abstract}

and pacing fields may be used to optimize transesophageal neurostimulation.

Keywords: spinal cord stimulation, transesophageal electrical pacing, transesophageal neurostimulation, esophageal catheter

https://doi.org/10.1515/cdbme-2019-0023

\section{Introduction}

The development of innovative types of esophageal electrodes or their further development is costly for a company. Additional costs are incurred for the time needed to produce prototypes and test them in a real environment, especially to test their interaction with biological tissue. Virtual simulations make it possible to create models with real material properties and to simulate and evaluate the interaction with their environment. This alternative can save costs and time.

Today the spinal cord stimulation (SCS) is one of the most common treatment methods for chronic pain [1]. The aim of the study was to model the spine and new esophageal electrodes for the transesophageal electrical pacing of the spinal cord [2].

Left ventricular heart stimulation in the esophagus is perceived as painful. Transesophageal neurostimulation attempts to inhibit the transmission of excitation through the nerves. This pain transmission is suppressed by emitting electrical impulses.

\section{Methods}

Offenburg, Badstrasse 24, D-77652 Offenburg, Germany, e-mail: mschleh@stud.hs-offenburg.de

Matthias Heinke: Biomedical Engineering Department of University of Applied Sciences Offenburg, Badstrasse 24, D77652 Offenburg, Germany, e-mail: matthias.heinke@hsoffenburg.de

Johannes Hörth: Biomedical Engineering Department of University of Applied Sciences Offenburg, Badstrasse 24, D77652 Offenburg, Germany, e-mail: johannes.hoerth@hsoffenburg.de
The modelling and simulation were carried out using the electromagnetic and thermal simulation software CST (Computer Simulation Technology, Darmstadt). The two esophageal electrodes were modeled on the basis of the technical manual of the temporary esophageal catheter TO8 from the manufacturer OSYPKA AG (Fig. 1). The thoracic 
spine was modeled based on the dimensions of a human skeleton [3].

The $20 \mathrm{~mm}$ esophageal balloon catheter and the thoracic spine were then integrated into the Offenburg heart rhythm model (Fig. 2). The simulation of the transesophageal neurostimulation is using the "Low Frequency Time Domain Solver" to show the electric field and the spread of electrical potential (Fig. 3 and 5).

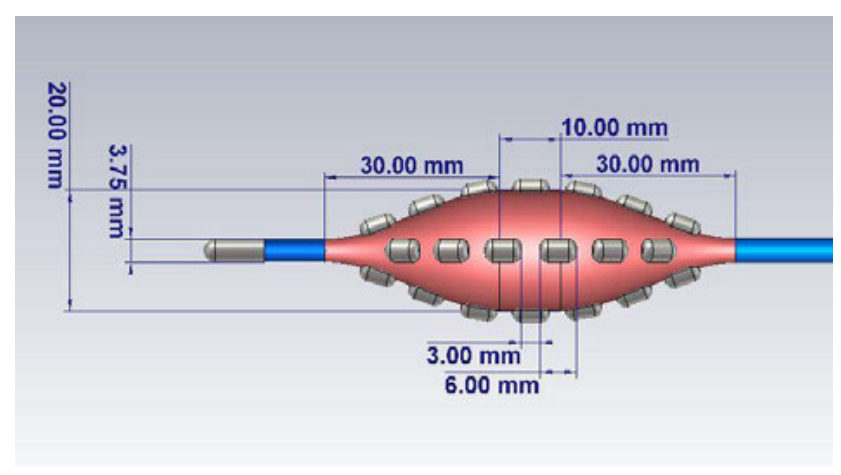

Figure 1: Esophageal balloon catheter

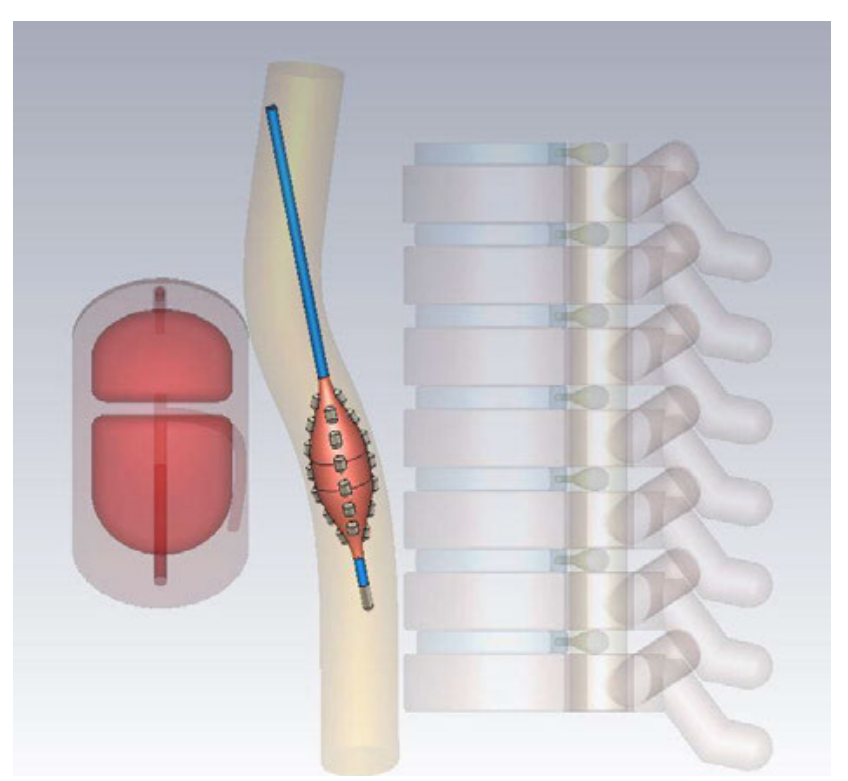

Figure 2: Esophageal balloon catheter and thoracic spine were integrated in the Offenburg heart rhythm model

\section{Results}

In addition, the transesophageal electrical pacing of the spinal cord was performed with a trapezoidal signal and an electric potential of $5 \mathrm{~V}$ assigned to the esophageal electrodes (Fig.4).
The neurostimulation was carried out for 1 second with a stimulation pulse of $0.01 \mathrm{~s}$ and a period of $0.05 \mathrm{~s}$. This corresponds to a frequency of $25 \mathrm{~Hz}$. The spread of the potential was measured at six points. After 1 second, a potential of $4.33 \mathrm{~V}$ could be measured directly at the electrode, $3.71 \mathrm{~V}$ at $2 \mathrm{~mm}$ into the myocardium, $2.68 \mathrm{~V}$ at $10 \mathrm{~mm}, 2.19 \mathrm{~V}$ at $30 \mathrm{~mm}$ and $2.1 \mathrm{~V}$ in the thoracic vertebra at a depth of $50 \mathrm{~mm}$. $2.08 \mathrm{~V}$ were measured in the spinal cord at a depth of $70 \mathrm{~mm}$ (Tab. 1).

The simulation was also carried out with $2 \mathrm{~V}$ and $7 \mathrm{~V}$.

Table 1: Comparison of the transesophageal neurostimulation

\begin{tabular}{llll}
\hline Depth of & $2 \mathrm{~V}$ & $5 \mathrm{~V}$ & $7 \mathrm{~V}$ \\
\hline $0 \mathrm{~mm}$ & $1.74 \mathrm{~V}$ & $4.33 \mathrm{~V}$ & $6.07 \mathrm{~V}$ \\
$2 \mathrm{~mm}$ & $1.48 \mathrm{~V}$ & $3.71 \mathrm{~V}$ & $5.19 \mathrm{~V}$ \\
$10 \mathrm{~mm}$ & $1.07 \mathrm{~V}$ & $2.68 \mathrm{~V}$ & $3.76 \mathrm{~V}$ \\
$30 \mathrm{~mm}$ & $0.88 \mathrm{~V}$ & $2.19 \mathrm{~V}$ & $3.07 \mathrm{~V}$ \\
$50 \mathrm{~mm}$ & $0.84 \mathrm{~V}$ & $2.10 \mathrm{~V}$ & $2.95 \mathrm{~V}$ \\
$70 \mathrm{~mm}$ & $0.83 \mathrm{~V}$ & $2.08 \mathrm{~V}$ & $2.91 \mathrm{~V}$ \\
\hline
\end{tabular}

These three passes show that the relation between the voltage delivered to the electrodes and the voltage applied to the spinal cord is linear.

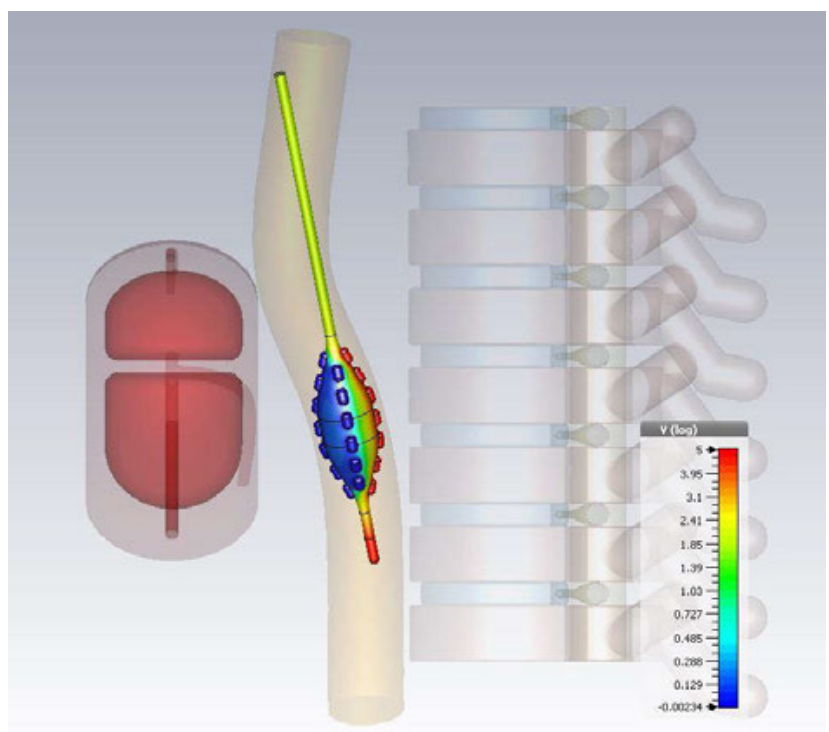

Figure 3: Esophageal balloon catheter with an electric potential at the electrodes for the transesophageal neurostimulation 


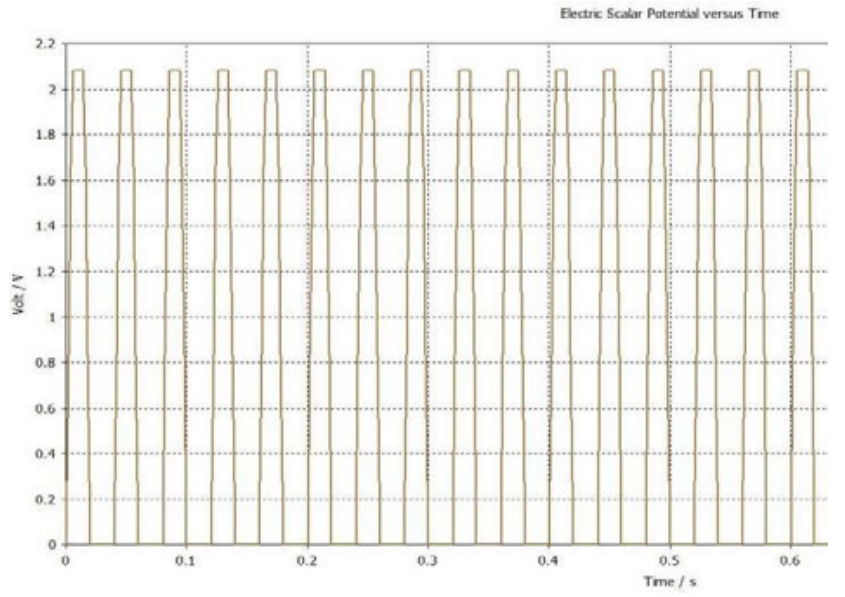

Figure 4: Measurement of spinal cord potential at $5 \mathrm{~V}$ during transesophageal neurostimulation
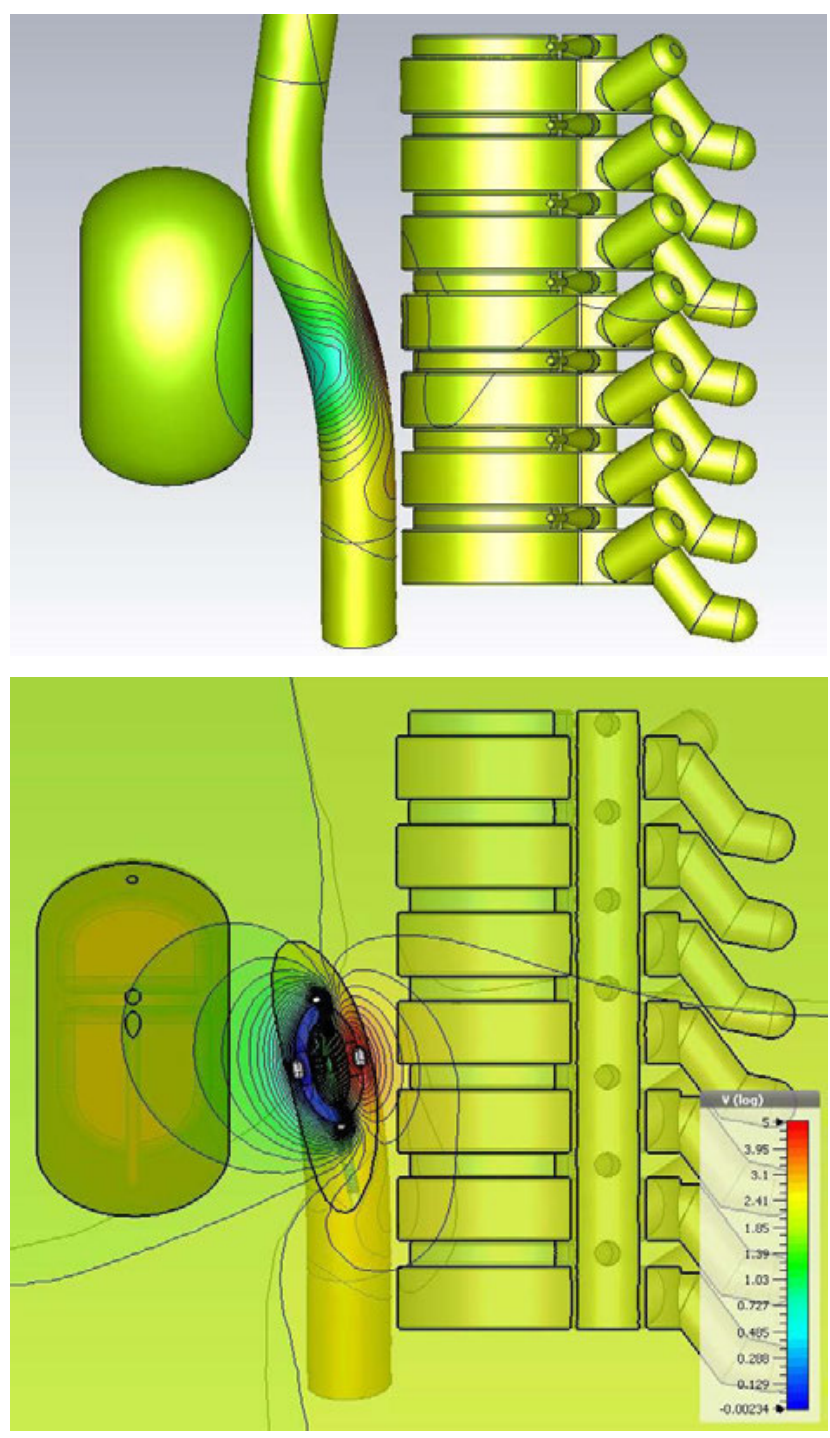

Figure 5: Potential distribution in transesophageal neurostimulation at 5V. Above: view from the outside. Bottom: view from the cutting plane

\section{Discussion}

Esophageal balloon catheter and heart rhythm models as well as the simulation of electrical pacing fields and electrical sensing fields allow the static and dynamic simulation of directed transesophageal electrical pacing of the spinal cord. The advantage of the simulation with CST is a realistic propagation of the electric field lines by selection of the different conduction properties of tissue and bones. The simulations show the electrical potential as well as the electric field lines detected in the spinal cord. The results of $25 \mathrm{~Hz}$ transesophageal neurostimulation provided positive results that could be used for further research in this topic. Thus, pain reduction during heart stimulation is possible. It becomes apparent that the transesophageal neurostimulation also spreads in the direction of the heart. Since the transesophageal neurostimulation is an unexplored procedure, its effects on cardiac stimulation are still unclear. The 3D simulation of the electrical sensing and pacing fields may be used to optimize transesophageal neurostimulation.

\section{Author Statement}

Research funding: The author state no funding involved. Conflict of interest: Authors state no conflict of interest. Informed consent: Informed consent has been obtained from all individuals included in this study. Ethical approval: The research related to human use complies with all the relevant national regulations, institutional policies and was performed in accordance with the tenets of the Helsinki Declaration, and has been approved by the authors' institutional review board or equivalent committee.

\section{References}

[1] Morales A, Yong RJ, Kaye AD, Urman RD: Spinal Cord Stimulation: Comparing Traditional Low-frequency Tonic Waveforms to Novel High Frequency and Burst Stimulation for the Treatment of Chronic Low Back Pain. Curr Pain Headache Rep. 2019 Mar 14;23(4):25. doi: 10.1007/s11916019-0763-3.

[2] Heinke M, Schalk M, „Ösophaguselektrodensonde und Vorrichtung zu kardiologischen Behandlung und/oder Diagnose“. München Patent DE102017010318B3, 2102 2019.

[3] Morgenstern U, Kraft M, Biomedizinische Technik Faszination, Einführung, Überblick, Bd. 1, Berlin: De Gruyter, 2014. 
